\title{
Patterns of food and nutrient intakes of Dutch adults according to intakes of total fat, saturated fatty acids, dietary fibre, and of fruit and vegetables
}

\author{
M. R. H. Löwik*, K. F. A. M. Hulshof and J. H. Brussaard \\ Department of Consumer Research and Epidemiology, TNO Nutrition and Food Research Institute, PO Box 360, \\ 3700 AJ Zeist, The Netherlands
}

\begin{abstract}
Dietary intake characteristics were studied among 3833 adults of the second Dutch National Food Consumption Survey held in 1992. The subjects were classified into three groups based on their intake of total fat (\% energy), saturated fatty acids (\% energy), dietary fibre (g/MJ), and fruit and vegetables $(\mathrm{g} / \mathrm{d})$. All the classifications resulted in differences in energy intake. Except for dietary fibre, the mean energy intake was higher in the higher-intake tertiles. For the classification based on total fat, saturated fatty acids and dietary fibre the more prudent diets were accompanied with a lower energy-intake. As to the consumption of food groups, differences existed in both the proportion of consumers and in the mean consumption among users. It is concluded that the trends observed are probably more important than the actual figures.
\end{abstract}

Dietary intake: The Netherlands

Nutritional standards for the Netherlands originate from two categories, namely the Recommended Dietary Allowances (RDA) with a long-standing tradition (Netherlands Food and Nutrition Council, 1992), and the Guidelines for a Healthy Diet, formulated in 1986 (Netherlands Food and Nutrition Council, 1986). In 1991 a reassessment of the goals related to fat consumption was carried out, and at this moment the Guidelines for a Healthy Diet are being updated. All the nutritional standards for the Netherlands are formulated by the Netherlands Food and Nutrition Council, an independent scientific advisory board for the Ministries of Health, Welfare and Sports and of Agriculture, Nature Management and Fisheries.

The Guidelines for a Healthy Diet can be summarized as follows: introduce variety into the diet; restrict the consumption of fat, notably saturated fat, and eat enough polyunsaturated fat; restrict the consumption of cholesterol; eat plenty of complex carbohydrates (starch) and fibre and avoid over-frequent and excessive consumption of sugar (both the monosaccharides and disaccharides); restrict alcohol consumption; and restrict the use of salt. In addition to observing these general rules, it is of course essential to achieve or maintain a normal body weight. These guidelines apply to the population as a whole; specific adjustments will be required for persons already on a diet.

In view of the population's current dietary habits, the Council recommends several changes in the nutritional composition of the diet. These changes apply to fat intake, vegetable/animal protein, cholesterol, (complex) carbohydrates, mono- and disaccharides, dietary fibre, alcohol and salt. For instance, the consumption of salt per individual should be such that it does not exceed the current daily average of $9 \mathrm{~g}$.
The relatively high fat intake observed for the Dutch population (Löwik et al. 1994), especially the intake of saturated fatty acids (SFA), is given the strongest emphasis in several policy statements because the scientific evidence of its relationship to health is strongest and the likely impact of a change in fat on public health is greatest (Van Wechem et al. 1998). Fat intake should be reduced from an average of $40 \%$ to $30 \%-35 \%$ of daily energy intake. This reduction should be brought about by limiting the amount of saturated fat consumed. In 1991 it was stated that total fat intake should be reduced to 30-35\%energy and that the intake of saturated fat should be reduced to $10 \%$ energy.

The data presented hereafter should be interpreted from the perspective of the above-mentioned standards.

\section{Methods}

In 1987-8, the first Dutch National Food Consumption Survey (DNFCS) was conducted (Löwik et al. 1994); the second DNFCS was carried out in 1992 (Löwik et al. 1998), whereas the data collection of the third survey was completed in March 1998. The first results of the third DNFCS are expected at the end of 1998. All surveys are conducted within the framework of the Dutch Nutrition Surveillance System (Löwik et al. 1996). Data were obtained from a probability sample of non-institutionalized subjects. Information on food consumption was collected with $2 \mathrm{~d}$ dietary records. In the dietary record method, respondents recorded their actual consumption of foods and beverages at the time of consumption. The amounts of the food products consumed were assessed by estimation of the weight of the food. In each household the person principally responsible 
for domestic affairs (main housekeeper) was the most important and was visited on two occasions by a specially trained dietitian. During the first visit the household diary was explained: in this diary all the food supplied by the main housekeeper to the household members as well as information on cooking methods, recipes and ingredients were recorded. The number of persons (including visitors) attending the different meals and the amounts of foods used by them, as well as amounts of leftovers and food given to pets, was noted. Household members (except children under 13) recorded food eaten outdoors in separate diaries. During the second visit the interviewers checked the diaries. Common household measures and food regularly used (i.e., slices of bread, amount of fat spread on bread, amounts of sugar added to tea and/or coffee) were weighed. All the information was used for the conversion of household data into intake figures on an individual level. To convert the food consumption data into dietary intake figures the Netherlands Food Composition Table was used. For this, the 1986-1987 version of the food composition table was used in the first DNFCS, and the 1993 version was used in the second DNFCS.

For the purpose of this country report, 3833 subjects 18-60 years of age (women had to be non-pregnant) of the second DNFCS, held in 1992, were selected. These subjects were classified into three groups (tertiles), whereby the classification was based on the subject's intake relative to the intake levels of the other subjects. This was carried out for total fat (\%energy), SFA (\%energy), dietary fibre $(\mathrm{g} / \mathrm{MJ})$, and fruit and vegetables $(\mathrm{g} / \mathrm{d})$. After this classification the mean intake of energy and nutrients and the average consumption of food groups was calculated for the separate tertiles. As to food groups, the proportion of users and the mean consumption among users was calculated.

\section{Results and discussion}

In Tables 1-5 the dietary intake figures for the various tertiles are presented. The results regarding the intake of dietary fibre should be interpreted with care, since the amount of dietary fibre refers mainly to insoluble fibre only. Mostly dietary fibre is defined as the combination of soluble and insoluble fibre.

In general, the characteristics of the diet were more in line with the guidelines for a healthy diet for the tertiles with a relatively low intake of total fat and of SFA and with a relatively high intake of dietary fibre and of fruit and vegetable consumption. As a matter of course, this is partly the result of the classification criteria in that the results are intentionally created in the direction of one of the goals of the guidelines.

All the classifications resulted in differences in energy intake. Except for dietary fibre, the mean energy intake was higher in the higher-intake tertiles. For the classification based on total fat, saturated fat and dietary fibre the 'better' diets were accompanied with a lower energy-intake. This may be (partly) the result of (selective) under-reporting, especially since it is known that under-reporting exists in the survey used for the calculations presented here. In that case, the 'better' diets are artificial in the sense that these diets are not realized in reality by the particular subjects. Therefore, the absolute level of the figures presented should be used with care. On the other hand, it should be realized that we used a large sample that was classified into tertiles, whereby large subgroups were created. Furthermore, mostly a clear trend or gradient was observed among the tertiles with the intermediate tertile having values between the lowest and the highest tertile. Therefore, it is most likely that the trend in the data is a valid observation, whereas the quantification of these trends may be biased by (selective) under-reporting. As to fruit and vegetables, an opposite trend regarding the intake of energy is observed. The mean energy intake was higher at a higher consumption level of fruit and vegetables. This is probably the result of the more general phenomenon that a higher consumption of a particular food group, in this case fruit and vegetables, is more likely to be realized by subjects with a higher energy requirement. For instance, the subjects with the highest fruit and vegetable consumption also had a higher mean consumption of meat and meat products and of cheese.

As to the consumption of food groups, differences existed both in the proportion of consumers and in the mean consumption among users. For the basic food groups, which are mostly used daily, the differences in the proportion of users were small if they existed at all. For these groups, with a proportion of users approaching $100 \%$, a more detailed classification is needed in order to come up with useable information. An extra argument for doing this is that nutritional advice often is to realize the guidelines by a substitution within product categories: for instance, choosing leaner varieties. The results of the first and second DNFCS show that the lowering of fat intake was indeed partly the result of the more frequent usage of leaner varieties (Hulshof et al. 1996).

In cases where an energy-providing nutrient is used to classify subjects into tertiles, in this case fat, the \%energy of the other macronutrients will increase when the energy obtained from fat decreases. The results show that fat is mainly 'substituted' by carbohydrates. Much smaller changes were observed for protein and for alcohol, probably partly as a consequence of the smaller amount of energy that is provided by these nutrients in general. Furthermore, for protein it is known that the \%energy is relatively stable, even in a cross-cultural setting.

A lower total fat intake (\%energy) is associated with a somewhat higher consumption of milk and milk products, whereas these products are among the major sources of fat. The reason for this apparent contradiction is that a lot of milk products have a \%energy below the mean \%energy observed for the population, whereby these products contribute to lowering the fat content of the diet as a whole. An opposite association was observed for SFA, in that a positive association was observed between the consumption of milk and milk products and the energy obtained from SFA.

In summary, the classification into tertiles regarding the intake of fat, SFA and to a lesser extent dietary fibre (due to methodological problems) and the consumption of fruit and vegetables provided relevant information. The trends observed are probably more important than the actual figures. 
Dutch patterns of food and nutrient intakes

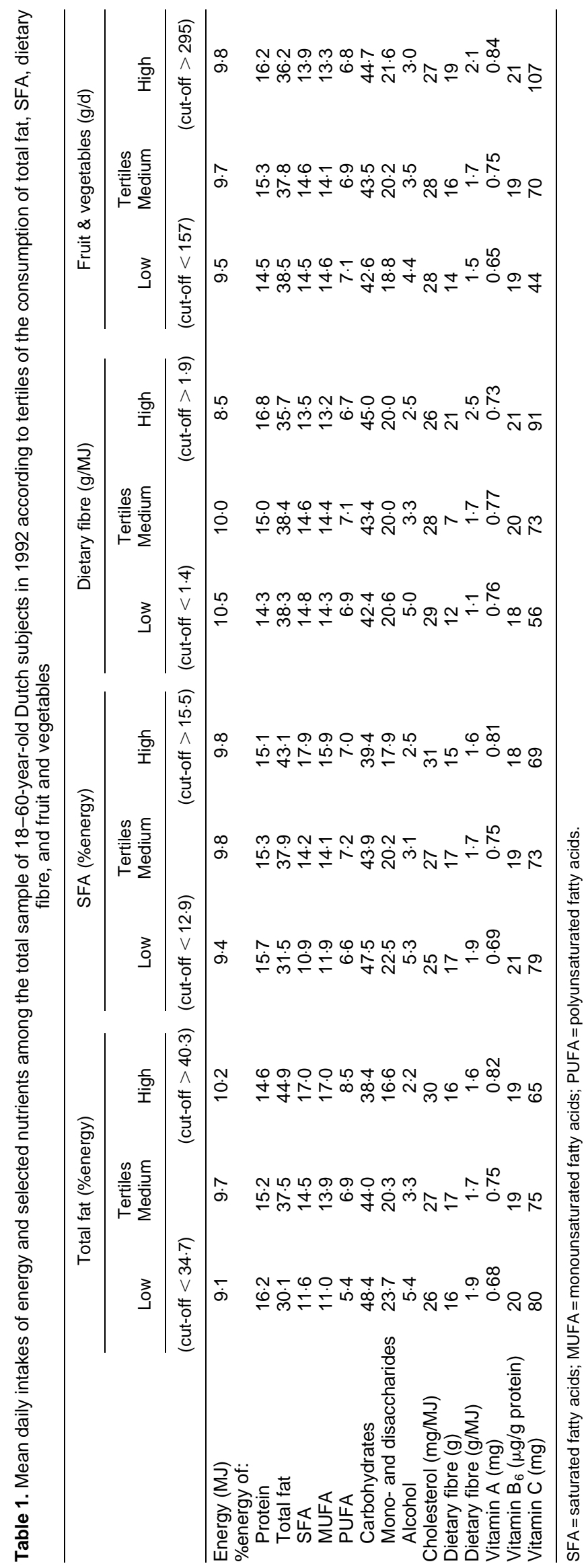







Dutch patterns of food and nutrient intakes

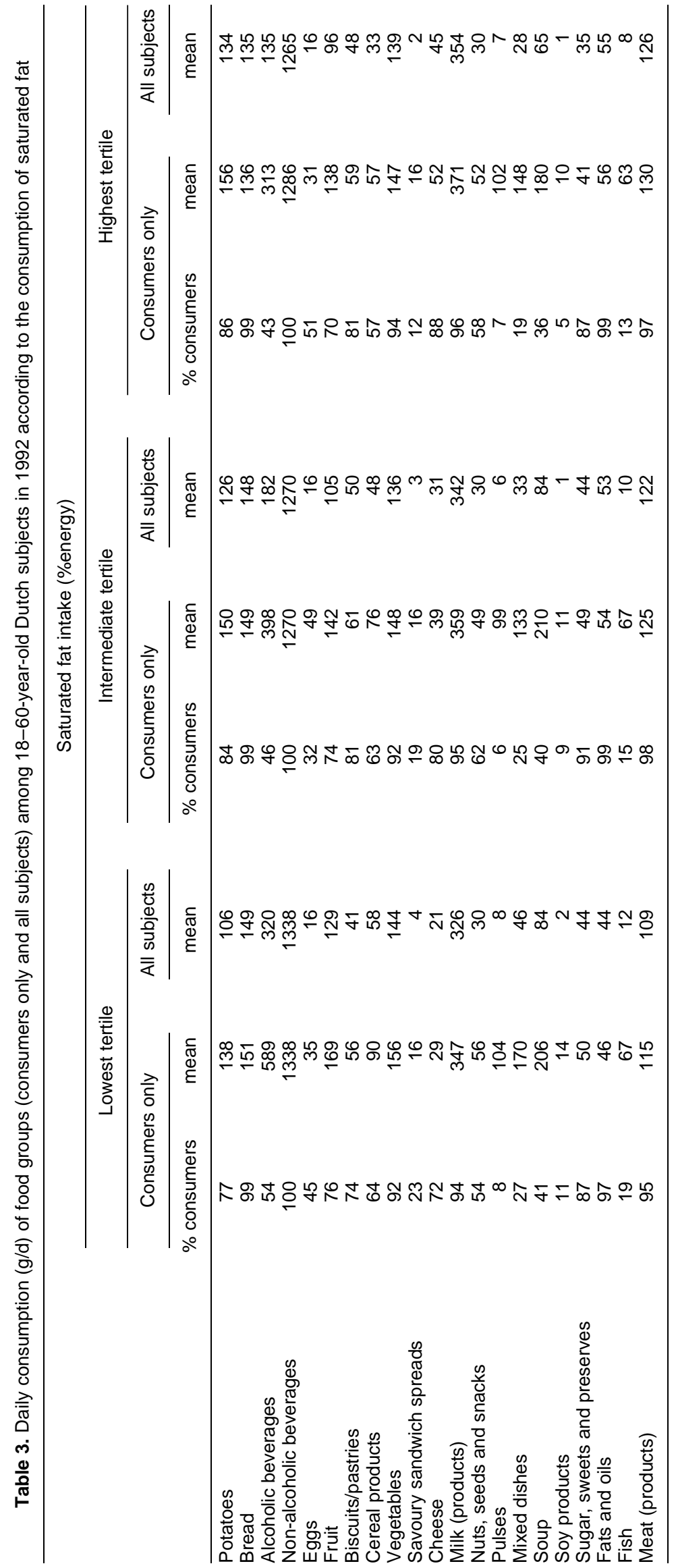




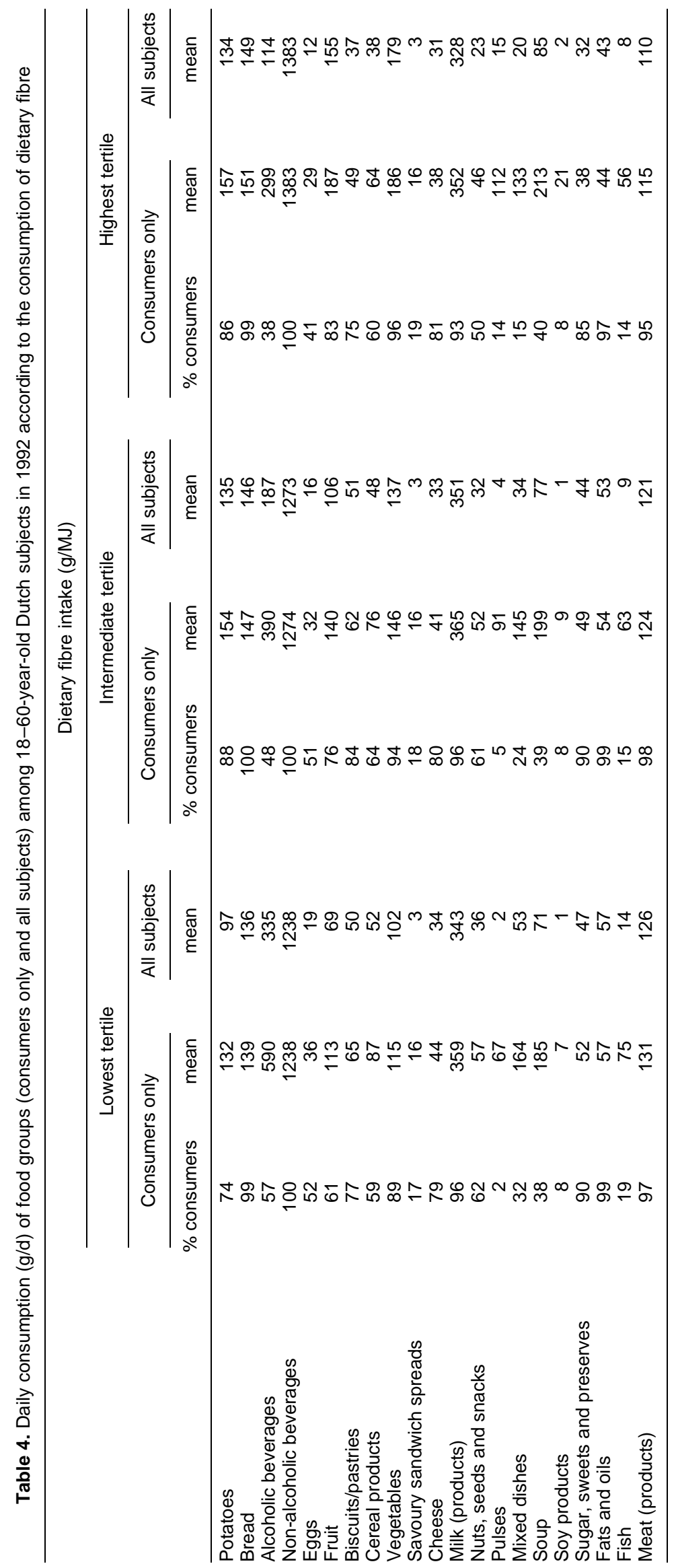


Dutch patterns of food and nutrient intakes

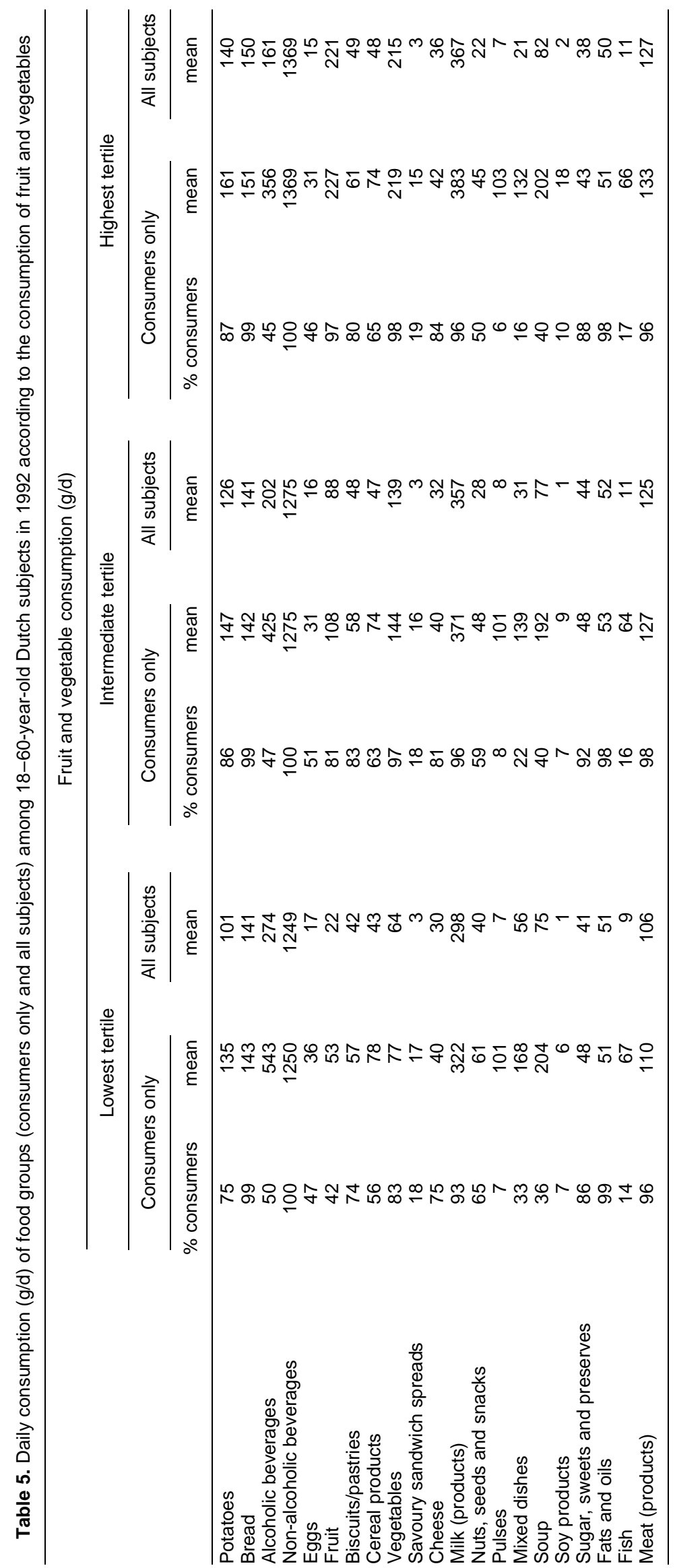




\section{References}

Hulshof KFAM, Beemster CJM, Westenbrink S \& Löwik MRH (1996) Reduction in fat intake in the Netherlands: the influence of food composition data. Food Chemistry 57(1), 67-70.

Löwik MRH, Brussaard JH, Hulshof KFAM, Kistemaker C, Schaafsma G, Ockhuizen Th \& Hermus RJJ (1994) Adequacy of the diet in the Netherlands in 1987-1988 (Dutch Nutrition Surveillance System) International Journal of Food Sciences and Nutrition 45 (suppl.1), S1-S62.

Löwik MRH, Hulshof KFAM, Brussaard JH \& Brants HAM (1996) Nutrition assessment and dietary guidelines: experience from the Dutch Nutrition Surveillance System. Proceedings of the Nutrition Society 55, 705-723.
Löwik MRH, Hulshof KFAM, Van der Heijden LJM, Brussaard JH, Burema J, Kistemaker C \& De Vries PJF (1998) Changes in the diet in the Netherlands 1987-1988 to 1992 (Dutch Nutrition Surveillance System). International Journal of Food Sciences and Nutrition 49 (suppl.1), S1-S64.

Netherlands Food and Nutrition Council (1986) Richlijnen goede voeding. Voeding 47, 159-181.

Netherlands Food and Nutrition Council (1992) Nederlandse voedingsnormen 1989. 2e druk. The Hague: Voorlichtingsbureau voor de voeding.

Van Wechem SN, Brug J, Van Assema P, Kistemaker C, Riedstra M \& Löwik MRH (1998) Fat watch: a nationwide campaign in the Netherlands to reduce fat intake - Effect evaluation. Nutrition and Health 12, 119-130. 